\author{
REVISTA ECONOMÍA \\ Vol. 72, N. ${ }^{\circ}$ II6 (noviembre 2020), I33-I42 \\ ISSN-i I390-6380, ISSN-e 2697-3332 | https://doi.org/10.29166/economia.v72i116.2585
}

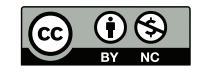

\title{
ACTIVACIÓN DE LOS CIRCUITOS ECONÓMICOS SOLIDARIOS: UN DESAFÍO DE LA POLÍTICA PÚBLICA DESDE EL PAGO DE BENEFICIOS SOCIALES EN ZONAS RURALES
}

\author{
ACTIVATION OF ECONOMIC SOLIDARITY CIRCUITS: A CHALLENGE OF PUBLIC \\ POLICY FROM THE PAYMENT OF SOCIAL BENEFITS IN RURAL AREAS
}

\author{
SERGIO EHIJOS MARDONES \\ Universidad de Aconcagua (Chile)
}

Recepción del manuscrito: 14 de septiembre de 2020

Aceptación versión final: 21 de noviembre de 2020

\begin{abstract}
RESUMEN Este artículo tiene por objeto analizar el impacto de las transferencias monetarias realizadas por el Estado en las economías locales y rurales, tomando como ejemplo a la región de La Araucanía. Partiendo de la base de lo importante que son estos recursos en la economía local y de las familias, hace una invitación a que el Estado incentive circuitos económicos en base a los principios de la economía social y solidaria, superando el mero asistencialismo que existe en el día de hoy.

palabras ClaVE Programas de bienestar; seguridad social; pensiones públicas; actividad económica regional; política pública.
\end{abstract}

ABSTRAC The purpose of this article is to analyze the impact of the monetary transfers made by the State on the local and rural economies, taking the region of La Araucanía as an example. Based on the importance of these resources in the local economy and in the families, it invites the State to encourage economics circuits based on the principles of the Social and Solidarity Economy, surpassing the mere assistance that exists today.

KEYWORDS Wellness programs; social security; public pensions; regional economic activity; public policy.

JEL CODES H53, H55, R11, R28.

\section{INTRODUCCIÓN}

La protección social en Chile involucra una serie de políticas y acciones públicas que buscan reducir el riesgo y la vulnerabilidad de tipo económico, así como intervenir para aliviar 
los casos de pobreza y de carencia extrema. La asistencia social, que se realiza bajo una óptica neoliberal, se ejecuta principalmente a través de transferencias monetarias públicas o programas de intervención social. El Ministerio de Desarrollo Social y Familia —en adelante, MINDES - tiene por misión.

Contribuir en el diseño y aplicación de políticas, planes y programas en materia de desarrollo social, especialmente aquellas destinadas a erradicar la pobreza y brindar protección social a las personas o grupos vulnerables, promoviendo la movilidad e integración social.

Por otro lado, la seguridad social constituye un derecho humano reconocido como tal en tratados internacionales y en las legislaciones naciones. En el mismo sentido, la Declaración Universal de los Derechos Humanos en su artículo 22 señala:

Toda persona como miembro de la sociedad tiene derecho a la Seguridad Social y a obtener, mediante el esfuerzo nacional y la cooperación internacional, habida cuenta de la organización y los recursos de cada estado, la satisfacción de los derechos económicos, sociales y culturales, indispensables a su dignidad y al libre desarrollo de su personalidad.

En la misma línea el artículo 19 de la Constitución chilena señala que la Constitución, en su numeral 18, asegura a todas las personas el derecho a la seguridad social. Posteriormente, la misma norma establece que: «La acción del Estado estará dirigida a garantizar el acceso de todos los habitantes al goce de prestaciones básicas uniformes, sea que se otorguen a través de instituciones públicas o privadas». Con respecto a lo que señala la Constitución, es importante aclarar que gran parte de los mecanismos de seguridad social en Chile se encuentran privatizados. Esto ocurre, por ejemplo, en el caso de las pensiones que funcionan en base a un sistema de capitalización individual que administran empresas privadas con fines de lucro llamadas Administradoras de Fondos de Pensiones. ${ }^{1}$ En este escenario, no se cumplen o se cumplen insuficientemente los principios de la seguridad social, ${ }^{2}$ lo que fue uno de los detonantes del estallido social de 2019. ${ }^{3}$ Es necesario recordar que el sistema de pensiones basado en la capitalización individual fue impuesto durante la dictadura militar de Augusto Pinochet y que a pesar de los esfuerzos de su Gobierno no todos los cotizantes del antiguo sistema de reparto optaron por cambiarse al sistema de capitalización, razón por la cual el sistema antiguo continúa funcionando. Debido a esto, en Chile existen dos sistemas de pensiones vigentes.

También es importante mencionar en este análisis que el Sistema Solidario de Pensiones, fue creado en el año 2008 a través de la ley 20.255; y que su finalidad es entregar pensiones no contributivas al $60 \%$ más vulnerable de la población mayor de 65 años. ${ }^{4}$

Tanto el sistema solidario como el de reparto son administrados en la actualidad por el Instituto de Previsión Social —en adelante, IPS—, que es un servicio público dependiente del Ministerio del Trabajo. Este servicio tiene por misión pagar mes a mes las pensiones y demás beneficios previsionales de ambos sistemas. A lo anterior se agrega que gran parte de los beneficios sociales otorgados por el Ministerio de Desarrollo Social se pagan a través del IPs. Se tiene entonces que la mayor parte de los mecanismos de transferencias monetarias del Estado por políticas de protección social y de lucha contra la pobreza, al igual que pensiones contributivas (estatales) y no contributivas son abonados mensualmente por el IPS a través de su red de pagos. Dentro de esta red, existe la modalidad de pagos presenciales en sectores rurales ${ }^{5}$ que se 
organizan en un día y en un horario previamente establecido de manera mensual. De esta manera, el Estado transfiere periódicamente una enorme cantidad de recursos a los territorios en forma de pensiones u otros beneficios sociales, que llegan directamente a las localidades, ya que estos beneficios se abonan, como se ha descrito, de manera presencial y en dinero en efectivo.

En este sentido, se constata en este artículo que el efecto práctico de estas trasferencias monetarias periódicas es el de crear una economía popular ${ }^{6}$ que puede en algunos casos derivar en economía solidaria en base a la maduración y articulación de las organizaciones sociales y sus diferentes niveles de cooperación. Para explicar la idea de la activación de un circuito económico solidario - en adelante, CES - sobre la coyuntura de los pagos rurales se presentan en este artículo dos casos distintos y se analiza cómo las organizaciones sociales pueden defender o no un lugar común. En un caso se podría hablar de un ces en gestación y en el otro solamente de un espacio común de economía popular.

Se analizaron en este trabajo dos ejemplos de la región de La Araucanía en Chile. La región es la tierra ancestral del pueblo mapuche, parte de lo que se conoce como Wallmapu, y que se mantuvo como territorio autónomo del Estado chileno hasta casi finales del siglo xix. Más del 60\% de las personas que se declaran mapuches en La Araucanía viven en zonas rurales. En su gran mayoría, son campesinos pobres. El 80\% de los productores mapuches posee una extensión de menos de 5 hectáreas de tierra. Por otro lado, La Araucanía es la región más pobre del país, y, por lo tanto, es la región en la que porcentualmente más beneficios sociales se pagan. Además, es la segunda con un mayor índice de ruralidad.? En este contexto, el pago de beneficios sociales que se realiza en localidades rurales es un importante acontecimiento, que en algunos lugares reúne a miles de personas. La presencia del pueblo mapuche con su lengua, sus tradiciones y sus costumbres da vida a lugares que por regla general son bastante calmos. ${ }^{8}$ Esto transforma a los pagos rurales de La Araucanía no solo en una actividad económica, sino que también en una instancia única de encuentro entre las familias y las comunidades. Esta particularidad social, cultural y económica de La Araucanía se debe tener en cuenta al momento de planificar políticas o de proyectar cambios que signifiquen una alteración en estos encuentros locales.

La pregunta de investigación que pretende responder este artículo es: ¿Pueden los pagos de beneficios en los sectores rurales activar un circuito económico solidario y de qué manera afectan a las economías locales los cambios en la forma como se entregan estos recursos?

Se parte de la hipótesis de que el pago de beneficios sociales y pensiones en sectores rurales puede ser aprovechado por el Estado para la creación de circuitos económicos solidarios y que la modificación de esta modalidad de pago, mediante el depósito de los beneficios en cuentas bancarias, por ejemplo, afecta gravemente la economía local de los territorios. Como segunda hipótesis, se sostiene que la organización social es condición indispensable para mantener una modalidad de pago y la economía que esta acción genera, ya que esta decisión es finalmente una política pública. Se pretendió realizar en este artículo un trabajo descriptivo usando el método cualitativo. Para ello, se utilizaron documentos oficiales, una entrevista en profundidad e información oficial recogida de las páginas web de las instituciones públicas vinculadas con la materia. También se revisaron las leyes y doctrina referidas a la seguridad social y al sistema de pensiones contributivo y no contributivo. 
Este artículo tiene, además, por objetivo analizar el impacto en la economía local de los pagos rurales, y de este modo plantear el desafío que significa para la administración pública, la activación de un circuito de economía solidaria. Se entiende aquí al ces como un:

[C]onjunto de relaciones económicas estructuradas como encadenamiento de flujos que tienden a ser recurrentes, y en este contexto se presentan como la esencia misma del proceso de circulación en el que se reconocen y definen las relaciones de comensalidad —compartir-, cooperación — trabajadores-, donación —organizaciones sin ánimo de lucro, organizaciones sociales, empresa privada, y Estado-, compensación o reciprocidad, tributación, intercambio —organizaciones sin ánimo de lucro, empresa privada y entidades crediticias- y de efectos redistributivos. (Lopera, 2009, p. 95)

\section{LOS PAGOS DE BENEFICIOS SOCIALES EN ZONAS RURALES Y EL ESTÍMULO ECONÓMICO «MONETARIO»A LAS PRÁCTICAS ECONÓMICAS LOCALES}

En la región de La Araucanía, el Instituto de Previsión Social tiene a su cargo la realización de aproximadamente 200.000 pagos mensuales, la mitad de los cuales corresponde a programas de protección social aplicados y diseñados por el MINDES, y la otra mitad corresponde a desembolsos por concepto de beneficios de seguridad social. Este pago de beneficios sociales representa en la región la no despreciable suma de 264.929.008 (miles de) pesos chilenos (IPS, 2019). El pago de estos beneficios se realiza principalmente bajo dos modalidades: presencial, en oficinas destinadas a estos efectos, o a través de una red de pagos móviles, mayoritariamente ubicadas en zonas rurales. De esta manera, el pago de los beneficios sociales llega a los lugares más apartados de la región. En 2019, los puntos de pago rurales sumaron 71 localidades. Una vez por mes se realiza el desembolso de estos beneficios, instancia en la que se congregan no solo los beneficiarios, sino que además familiares o acompañantes, comerciantes, funcionarios públicos, etc. Éste es un escenario ideal para la formación de ferias libres y para la realización de actividades comerciales que generan una «economía popular» que tendrá mayor o menor envergadura dependiendo de la cantidad de beneficios pagados en el sector. El desembolso de estos beneficios se realiza en dinero en efectivo que se cuenta — en voz alta - y se entrega en detalle por parte del equipo pagador. Como comenta Samuel Soto Villegas, quién es funcionario del Instituto de Previsión Social y tiene dentro de sus funciones la de supervisar el desarrollo de los pagos rurales (Soto, 2020), «normalmente se realiza una fila en la cual se identifica al beneficiario o su apoderado y luego de esto se procede al pago de los beneficios con dinero en efectivo».

Esta modalidad de pago rural (o móvil) fue muy utilizada hace un par de décadas, sin embargo, ha ido perdiendo terreno principalmente por el avance de la red de pago presencial, por el cambio de las normativas de seguridad y transporte de valores y por el uso de nuevas tecnologías, como el acceso masivo a tarjetas de débito. En este último caso, se tiene a la Cuentarut del Banco del Estado, que permite el depósito directo de los beneficios monetarios en una tarjeta de cuenta vista. ${ }^{9}$ Esta última opción, debido a sus bajos costos, es la preferida por las instituciones del Estado. 
A pesar de los cambios descritos, los pagos rurales en la región de La Araucanía representan la suma de 1.255.347.430 (miles de millones de) pesos chilenos por mes ${ }^{10}$ que se efectúa a 11.972 beneficiarios. Cabe destacar que algunos puntos, de los 71 de la región, concentran una mayor cantidad de pagos, por lo que significan un importante incentivo para las economías locales. La transferencia monetaria realizada desde el Estado constituye un evento mensual que representa una enorme oportunidad para los comerciantes y los productores agrícolas locales. Esta transferencia no solo beneficia a las ferias y vendedores ambulantes, sino que además es un fuerte estímulo para los comerciantes establecidos y la realización de actividades de servicios e incluso de turismo.

También se puede destacar el hecho de que fuera de la actividad monetaria, se produce una activación no monetaria que se traduce en un importante número de encuentros familiares, reuniones sociales, comidas, actividad de grupos sociales como clubes de adultos mayores, pensionados, clubes deportivos e incluso actividades religiosas. Esto transforma el día de pago de beneficios en una verdadera fiesta popular que da vida a las 71 localidades visitadas.

\section{LOS PAGOS RURALES COMO UN ACTIVADOR DE CIRCUITO DE ECONOMÍA SOLIDARIA}

Como ya se expuso anteriormente, la opción de pago preferida por el Estado es la del traspaso de los beneficios a una cuenta vista que se opera a través de una tarjeta de débito. Esta opción es la más barata y cómoda para el Estado, pero representa, sin embargo, un problema para las personas que no están acostumbradas a estas tecnologías y especialmente para los adultos mayores. Por otro lado, el traspaso masivo de pagos de beneficios desde la modalidad móvil o rural, a tarjeta de débito, perjudica la realización de las ferias libres y las otras actividades comerciales que se relacionan con el día de pago.

Una vez descrito el imparto económico y social del pago de beneficios en la modalidad de pago móvil, este artículo busca reparar sobre el hecho de que esta actividad periódica de transferencias monetarias no ha sido pensada desde el Estado para cumplir con otros fines, como la de generar desarrollo económico local. Desde la perspectiva de la economía social y solidaria, este importante traspaso de recursos monetarios se podría pensar como un activador de un circuito de economía solidaria que genere desarrollo sostenible en los territorios y que involucre la participación colaborativa entre los habitantes de los sectores. En ese sentido, el Estado no se ha propuesto dejar atrás un modelo asistencialista que crea dependencia en los territorios, desarticula el tejido social y muchas veces crea una competencia salvaje entre los comerciantes. En la mayoría de los casos, se observa una reproducción del modelo económico neoliberal y sus lógicas a escala local.

El Estado central o municipal podría promover la actividad y la organización de los productores locales, de cooperativas o de asociaciones de productores, vendedores o consumidores. El Estado, como se ha mostrado, ya interviene en las localidades al llevar directamente el pago de los beneficios, sin embargo, muchas veces esos recursos vuelven a las ciudades, ya que los beneficiarios necesitan proveerse de bienes de consumo, muchos de los cuales perfectamente podrían ser ofrecidos localmente. En este sentido se estima, al igual que Luz Lopera (2009), que: 
La articulación de las empresas, organizaciones de economías solidarias entre sí y con las instituciones públicas y privadas, auxiliares del sector [...] generan mercados que constituyen circuitos económicos solidarios o agrupamientos —en el lenguaje empresarial- que desarrollan espacios comunitarios para realizar las actividades y vocaciones económicas y sociales de las diferentes comunidades y de sus estamentos sociales. (p. 92)

Para los fines de activar un CEs, la promoción y el apoyo del Estado es muy importante, pero también la maduración de las organizaciones locales puede dar paso a mayores grados de solidaridad lo que puede conducir a crear un tipo diferente de desarrollo basado en la articulación de un CES.

\section{DIFERENTES GRADOS DE MADURACIÓN DE LAS ORGANIZACIONES SOCIALES. EL ANÁLISIS COMPARADO DE LAS FERIAS LIBRES DE LAS LOCALIDADES DE LUMACO Y QUEPE}

Para aterrizar los conceptos expuestos en este artículo, se puede analizar brevemente el caso de dos puntos de pagos, que podrían definirse como «grandes» en relación con el número de beneficiarios, y en cuyo caso las organizaciones sociales no han mostrado el mismo grado de maduración.

\section{CASO DE LUMACO}

Esta localidad se encuentra a 120 kilómetros de Temuco la capital regional de La Araucanía. Hasta el mes de abril de 2016, los pagos de esta ciudad se realizaron bajo la figura de pago móvil, esto es, un equipo pagador visitaba la ciudad un día y en un horario determinado y procedía a pagar los beneficios en dinero efectivo. Sin embargo, a partir del mes de mayo del mismo año, se encargó la realización de estos pagos al Banco del Estado a través de su nueva sucursal en dicha ciudad. A partir de entonces, el pago no se realizaba en un solo día, sino que se distribuía durante todo el mes. Esto, que en principio era muy bueno para los pensionados, ya que no tenían que hacer filas y esperar largo rato para recibir sus pagos, significaba también la eliminación de la feria libre que se instalaba aprovechando la coyuntura. Los feriantes, en su mayoría mapuches y productores locales, protestaron por esta medida ante las autoridades locales y el IPS. Ellos estaban organizados en la agrupación de feriantes Newen Mapu que incluso contaba con personalidad jurídica.

La organización que logró el respaldo de la municipalidad de Lumaco luchó varios meses por revertir la medida. Luego de muchas reuniones y conversaciones (Soto, 2020), «muchas de ellas muy tensas y complicadas», se logró finalmente un acuerdo por el cual se mantenían los pagos en la oficina del Banco del Estado de Lumaco, pero se concentraban de manera de lograr que la situación fuera bastante similar a la que existía antes. La municipalidad de Lumaco, que también participó de este acuerdo, se comprometió a cerrar las calles el día de la feria y dar facilidades para la instalación de los comerciantes. En el mes de noviembre de 2016 se reinauguró la feria libre de Lumaco que sigue dándole trabajo a 80 productores locales. La organización y la solidaridad entre los feriantes fue fundamental para revertir una política de gobierno que en principio no pensaba en la importancia de mantener la feria libre como un espacio de activación económica y de encuentro social. Manuel Curín, presidente de la agrupación de 
feriantes Newen Mapu, comentó: «Estoy muy agradecido y contento de volver, porque somos una feria que desde 1985 se realiza y estos meses detenidos fueron difíciles para nosotros, ahora solo esperar que la gente nos acompañe este lunes 14 de noviembre»; ${ }^{11}$ el mismo Curín señaló que «ha vuelto a nuestro pueblo la alegría, la tradición y el reencuentro familiar, esto da vida a Lumaco». ${ }^{12}$ En este caso, se puede comprobar, como dice Razeto (2005), que «la unión de conciencias, voluntades y sentimientos tras un objetivo compartido genera una energía social que se manifiesta eficientemente, dando lugar a efectos positivos e incrementando el logro de los objetivos de la organización en que opera» (p. 972).

\section{CASO DE QUEPE}

Esta localidad se encuentra a 16 kilómetros de Temuco. Por tratarse de un pago móvil o rural, una vez por mes se realiza el desembolso de beneficios a cargo del Instituto de Previsión Social. Al igual que Lumaco, se puede clasificar esta localidad como un "pago grande», ya que reúne un importante número de beneficiarios. Tal como en Lumaco, el día de pagos se realiza una feria y se junta gran cantidad de gente, (Soto, 2020): «[L]as personas que llegan se cuentan en miles, ya que generalmente los beneficiarios llegan acompañados de sus familiares. A eso se deben agregar los feriantes y otros comerciantes que aprovechan el día». Sin embargo, la mayoría de los comerciantes no son de la localidad y los productos que se venden no son de productores locales. Tal como comenta Samuel Soto (2020), «la gran mayoría de los comerciantes son de afuera, se movilizan por toda la región siguiendo los días de pago». Otra característica importante es que los feriantes que realizan sus actividades el día de pago de beneficios, no se encuentran organizados. Según explica Soto (2020), los feriantes de Quepe «nunca han estado organizados, hay una feriante que a veces habla por todos, pero nunca he sabido de que existan reuniones periódicas o alguna directiva». Según Soto:

[A]lgunas veces se han hecho algunos cambios relacionados con los lugares de pago o los días determinados. Otras veces hemos tenido algunas contingencias o algunas modificaciones. Llama la atención que no exista una organización social de los pensionados o de los propios feriantes, que nos pregunte qué es lo que pasa.

Por otro lado, de acuerdo con lo que explica el funcionario, existen muchas actividades que organiza su servicio público o la municipalidad local, aprovechando el día de pagos, (Soto, 2020):

[P]or lo general, se hacen actividades como operativos médicos, visita de servicios públicos, actividades de gobierno en terreno, etc., las que no se planifican con ninguna organización social local. Solo se les avisa o se les comunica por afiches o por medios radiales, de eso se encarga la autoridad municipal o algunas veces los servicios que organizan el operativo.

El análisis de estos casos muestra que la actividad comercial y la periodicidad de los pagos y la actividad económica que conlleva, no necesariamente cuajan en organización social. En el caso de Lumaco, la realización de la feria mensual llevó a sus miembros a unirse y a crear una asociación de comerciantes legalizada y reconocida como interlocutora válida por las autoridades locales y centrales. Eso ayudó a los feriantes a defender su fuente de trabajo y a conseguir beneficios adicionales por parte del Estado. En el segundo caso, el camino ha sido diferente, 
ya que la actividad de la feria mensual no ha llevado a la organización de los comerciantes ni de otros grupos sociales como los productores o los beneficiarios de los beneficios estatales. Como se observa en este análisis, en Quepe las actividades de los servicios públicos y de las autoridades locales no se comentan ni se planifican con la sociedad civil, ya que ésta no se encuentra organizada; esto, a diferencia de lo que ocurre en Lumaco. Para el caso de Quepe, valdría la pregunta: ¿Qué pasaría si el Estado decide reemplazar la modalidad de pago rural y concentrado en dicha localidad? A la luz de los antecedentes descritos en este artículo, lo más probable es que nada. Esta opinión se confirma con la experiencia de los cambios de la modalidad de pagos de las ciudades de Teodoro Schmidt (2016) y Curarrehue (2018), ambos muy similares a la localidad de Quepe.

\section{CONCLUSIÓN}

En Chile, los mecanismos de protección social y de seguridad social tienen como fin reducir el riesgo y la vulnerabilidad económica. Los dispositivos de seguridad social establecen ciertas técnicas de protección de contingencias, que tienen el rasgo característico de que han sido encargadas de manera significativa a la empresa privada. Sin embargo, el Estado a través del Instituto de Previsión Social se encarga del pago de los beneficios del Sistema Previsional de Reparto o «antiguo» y del Sistema Solidario, a lo que se suma la responsabilidad de la realización del pago de beneficios de los programas de protección social dependientes del Ministerio de Desarrollo Social y Familia. Esto hace que el IPs sea clave en la transferencia de recursos desde el Estado hacia las personas y zonas que más lo necesitan.

Esta transferencia monetaria se realiza muchas veces en sectores rurales como traspaso de dinero en efectivo en un día y una hora determinada. El pago de beneficios es aprovechado de manera desorganizada por diferentes grupos sociales como los comerciantes y los feriantes. Sin embargo, esta actividad que concentra miles de personas en algunas localidades y que significa el traspaso de enormes recursos monetarios, no ha cuajado necesariamente en organización social o en entidades de economía solidaria. Por otro lado, y respondiendo a la pregunta de investigación formulada en la introducción, el Estado, pudiendo hacerlo, no ha aprovechado la oportunidad que esta transferencia significa para crear mecanismos de desarrollo durables a través del fortalecimiento de las asociaciones de productores, incentivo de la creación de cooperativas de productores vendedores y consumidores, etc.; de modo de activar procesos locales y fortalecer el tejido social de protección social. La lógica neoliberal que ha imperado en los últimos 40 años es la del asistencialismo que inyecta recursos en los sectores más pobres pensando en el consumo, pero dejando de lado otras posibilidades como el desarrollo económico local.

En este sentido, la coyuntura del pago de beneficios sociales en sectores rurales, que es la ocasión ideal para la activación de un circuito económico solidario por parte del Estado, ha sido desaprovechada. Por otra parte, se confirma la primera hipótesis sugerida en este artículo en cuanto a que el cambio de la modalidad de pago presencial en sectores rurales afecta de manera significativa a la economía local, lo que se expresa primeramente en la desaparición de las ferias. Por otra parte, y confirmando lo señalado en la segunda hipótesis, la existencia de 
organizaciones sociales es condición fundamental para defender el espacio común creado en los territorios con ocasión del pago de beneficios. Queda pendiente como un desarrollo posterior de esta investigación un análisis cuantitativo del impacto económico que sígnica la realización de los pagos rurales en las diferentes localidades de la región.

Reafirmando las ideas presentadas en este artículo, se estima que otro tipo de desarrollo es posible. El encuentro entre los productores locales con los consumidores locales y la permanencia en los territorios de los recursos trasferidos por el Estado como beneficios sociales pueden generar un desarrollo sustentable. Si bien existe la oportunidad de que estas transferencias monetarias puedan activar un circuito económico solidario, existe por otro lado, una alta probabilidad de que estos recursos se sigan concentrando y se elimine la posibilidad de crear un circuito de producción y de consumo alternativo que tenga como base a los productores y los consumidores locales.

Como se ha visto en este artículo con los pagos rurales y la intervención que ello significa, se genera una activación desde lo monetario que forma una economía popular. Sin embargo, esto no deriva necesariamente en la formación o en el fortalecimiento de redes o en entidades de economía social y solidaria autónomas y sostenibles, debido a que no se miran ni se activan procesos de articulación donde el factor c de la asociatividad y la colaboración opere a lo largo de todo el proceso de producción, distribución y consumo, junto a mecanismos de comunicación, de educación y de finanzas que concuerden con la misma racionalidad. La creación de un vínculo virtuoso entre productores, consumidores locales y mecanismos de fomento, en clave solidaria, permitiría la activación de un circuito económico solidario gestado desde los territorios aprovechando la coyuntura del pago de beneficios sociales en los sectores rurales. Lo anterior es posible, solo se trata de voluntad, compromiso y de imaginación.

\section{NOTAS}

1 Un análisis detallado de las falencias del Sistema de Capitalización Individual se lo puede encontrar en la obra Aquí se fabrican pobres: el sistema privado de pensiones chileno, de Carlos Rivadeneira, en especial en las páginas 95 a 187.

2 Mayores antecedentes sobre los principios de la seguridad social se pueden encontrar en la obra Derecho del trabajo y de la seguridad social, de H. Humeres Magnan y H. Humeres Noguer, pp. 457-462. 3 La tasa de reemplazo promedio en Chile es de 34\% — dato obtenido de la Fundación Fiel-Chile-. Por otro lado, existe una gran diferencia entre las pensiones autofinanciadas de los hombres y las mujeres en desmedro de estas últimas. En las conclusiones de la Comisión Asesora Presidencial para la reforma del Sistema de Pensiones o Comisión Bravo, se señala que «un 50\% de los pensionados entre los años 2025 y 2035 obtendrían tasas de reemplazo igual o inferior al 15\% del ingreso promedio de los últimos años». 4 Mayores antecedentes sobre el sistema solidario de pensiones se pueden encontrar en la obra de Hugo Cifuentes Lillo, El sistema de seguridad social chileno, regímenes no contributivos de pensiones.

5 Otra forma de pago es el depósito en cuenta bancaria o el pago en oficina bancaria o licitada especialmente para estos efectos. En este trabajo, solo se analiza la modalidad de pago presencial rural.

6 «Es la economía empírica de les trabajadores, dependientes o autónomos, de les que viven o quieren vivir de su trabajo, es la economía de sus familias, comunidades, asociaciones, organizaciones y redes de cooperación o ayuda mutua, formales o informales» (Coraggio, 2020, p. 12).

7 Datos sacados del Plan Impulso Araucanía.

8 En muchos sectores rurales, la actividad económica y de intercambios se realiza únicamente con 
ocasión del pago de beneficios sociales. Esto debido principalmente a la instalación de ferias itinerantes. 9 La Cuentarut es una cuenta vista que incluye una tarjeta de débito. El número de la Cuentarut es el número de rol único tributario (RUT) de cada usuario sin su dígito verificador. De esta manera todas las personas que tengan un RUT chileno - mayores de 12 años las mujeres y 14 años los hombres- pueden optar a este servicio. En la cuenta pública 2018, se señala que en ese año el total de Cuentarut fue de 11,2 millones. Cabe recordar que la población chilena se estima en algo más de 18 millones de personas. 10 Datos a 2019.

11 Nota de prensa de la municipalidad de Lumaco.

12 Carta de agradecimiento de fecha 17 de noviembre de 2016.

\section{REFERENCIAS}

Cifuentes, H. (2018). El sistema de seguridad social chileno. Ediciones UC.

Constitución Política de la República de Chile. (2020). Biblioteca del Congreso Nacional. http://bcn. $\mathrm{cl} / 2 \mathrm{f} 6 \mathrm{sk}$.

Coraggio, J. L. (2020). Economía social y economía popular: conceptos básicos. Consejo Consultivo INAES. https://www.coraggioeconomia.org/jlc/archivos\%2opara\%2odescargar/Economia\%2oSocial\%20 y\%20Economia\%2oPopular\%20-\%2oConceptos\%2oBasicos.pdf

Humeres Magnan, H. y Humeres Noguer, H. (1992) Derecho del trabajo y la seguridad social. Editorial Jurídica de Chile.

Informe Consejo Asesor Presidencial para la Reforma del Sistema Previsional. (2006). Previsión Social. https://www.previsionsocial.gob.cl/sps/download/estudios-previsionales/comisionpensiones/ documentos-interes-general/informe-consejo-asesor-presidencial-reforma-del-sistema-previsional-comision-marcel-2006.pdf última visita 24 de agosto de 2020. Chile.

Informe final. (2015). Comisión Asesora Presidencial sobre el Sistema de Pensiones. http://www.comision-pensiones.cl/Informe_final_CP_2015.pdf

Instituto de Previsión Social (IPS). (2019). Cuenta Pública. https://www.ips.gob.cl/servlet/internet/archivo/1421810867610/libro_cuenta_publica_2019.pdf

Instituto de Previsión Social (IPS). (2020). Estadísticas. https://www.ips.gob.cl/servlet/internet/inicio/ conozcanos/estadisticas-infografias

Lopera, L. (2009). Los Circuitos económicos solidarios: espacios de relaciones y consensos. Semestre Económico, 12(25), 81-93.

Ministerio de Desarrollo Social y Familia (MINDEs). (2020). Misión institucional. http://www.desarrollosocialyfamilia.gob.cl/mision

Municipalidad de Lumaco. (2016). Nota de prensa. https://www.munilumaco.cl/index.php/ noticias-home/342-pago-d-epensiones

Naciones Unidas. (2020). Declaración Universal de los Derechos Humanos. Documentos. https://www. un.org/es/documents/udhr/UDHR_booklet_SP_web.pdf

Plan Impulso Araucanía. (2019). Contexto. https://planimpulso.cl/wp-content/uploads/2019/12/Plan_ impulso_araucania.pdf

Razeto, L. (2005). El concepto «solidaridad». Pensamiento Crítico Latinoamericano, 2, 971-985.

Rivadeneira, C. (2017). Aquí se fabrican pobres: el sistema privado de pensiones chileno. Lom Ediciones.

Soto, S. (2020). Comunicación personal. 24 de agosto de 2020. 